\title{
Revisão Sistemática de Literatura sobre o Pensamento Computacional por Meio de Objetos de Aprendizagem
}

\author{
Joethe Moraes de Carvalho ${ }^{1}$, José Francisco de Magalhães Netto ${ }^{2}$, Thais Oliveira \\ Almeida ${ }^{2,3}$ \\ ${ }^{1}$ Departamento de Ensino, Pesquisa e Extensão - Instituto Federal de Educação, Ciência e \\ Tecnologia do Amazonas (IFAM) - 69.190-000 - Maués - AM - Brasil \\ ${ }^{2}$ Programa de Pós-Graduação em Informática (PPGI) - Universidade Federal do Amazonas \\ (UFAM) - 69.067-005 - Manaus - AM - Brasil \\ ${ }^{3}$ Departamento de Ciência da Computação - Universidade Federal de Roraima (UFRR) - \\ 69.310-000 - Boa Vista - RR - Brasil \\ joethe@hotmail.com, jnetto@icomp.ufam.edu.br, thais.oliveira@ufrr.br
}

\begin{abstract}
Computational Thinking (CT), which is application of computer science techniques to solve problems in most diverse areas of knowledge, has grown moderately in the Brazilian Educational Scenario. To aid in the use of these techniques, educational tools known as Learning Objects (LOs) are used. This article aims to carry out survey of activities based on the CT to identify and analyze existing tools. This work presents a Systematic Review of Literature (SRL) composed of 39 articles published on this topic, in the most relevant digital repositories on Informatics in Education, since the year 2012. The results obtained show the most usual actions about this growing area, as well as the instruments that support them, with a view to encouraging futures and developing works.
\end{abstract}

Resumo. O Pensamento Computacional (PC), que é a aplicação de técnicas de Ciências da Computação para resolução de problemas nas mais diversas áreas do conhecimento, tem crescido moderadamente no cenário educacional brasileiro. Para auxiliar no uso dessas técnicas, são usadas ferramentas educativas conhecidas como Objetos de Aprendizagem (OAs). Este artigo tem por objetivo realizar o levantamento das atividades embasadas pelo PC para identificar e analisar as ferramentas existentes. Para isso, este trabalho apresenta uma Revisão Sistemática de Literatura (RSL) composta de 39 artigos publicados sobre esse tema, nos repositórios digitais mais relevantes sobre Informática na Educação, desde o ano de 2012. Os resultados obtidos mostram as ações mais usuais acerca dessa área em ascensão, bem como os instrumentos que as apoiam, visando favorecer trabalhos em desenvolvimento e futuros.

\section{Introdução}

As tecnologias nos cercam em todos aspectos de nossas vidas. Na área da educação, a cada ano, muitas inovações surgem, aprimorando técnicas que visam facilitar o processo de ensinoaprendizagem. Podemos citar como exemplo dessas novidades, softwares de computador e aplicativos para dispositivos móveis, voltados para a promoção do desenvolvimento do 
intelecto dos discentes. Esses instrumentos mostram-se fomentadores da evolução perceptiva dos alunos e tornam-se aliados em potencial dos educadores.

Essas ferramentas educativas são conhecidas como Objetos de Aprendizagem (OAs) e, segundo [Braga \& Menezes 2014], se caracterizam principalmente pela reusabilidade em diferentes contextos e funcionam como mediadores do conhecimento. As autoras acrescentam ainda que, quando bem utilizados, os OAs tornam-se grandes aliados ao processo educativo e podem estimular a aprendizagem nos discentes.

Outro método que gradativamente tem tomado espaço no ambiente educacional brasileiro é o Pensamento Computacional (PC), que foi proposto por [Wing 2006] e consiste em resolver problemas nas diversas áreas do conhecimento utilizando os conceitos de Ciências da Computação. A autora ainda incentiva que essas habilidades sejam ensinadas às crianças, assim como as habilidades de aritmética, leitura e escrita. Diante disso, surge a seguinte questão de pesquisa: quais seriam as práticas embasadas pelo $\mathrm{PC}$ e quais os OAs que vêm sendo empregados para este fim?

Os estudos dessas ações no campo da Informática na Educação são fundamentais para acompanhar os processos em constante evolução, visando não apenas manter-nos atualizados acerca dessas inovações, mas para que os profissionais envolvidos na esfera da educação, como educadores e pesquisadores, possam aplicá-las, buscando a melhoria do ensino em nosso país. Para responder à questão de pesquisa, este artigo tem por objetivo realizar o levantamento das atividades embasadas pelo PC, para identificar e analisar as ferramentas existentes no âmbito educacional.

Para isto, este trabalho apresenta uma Revisão Sistemática de Literatura (RSL), que é um processo de investigação, e busca identificar, selecionar, avaliar e sintetizar os materiais produzidos sobre um tema, visando responder a uma questão bem definida. Foram consideradas as principais pesquisas relacionadas com o PC no meio educacional nos últimos 5 anos. As metas foram definidas pelo protocolo Goal-Question-Metric (GQM), que é um instrumento para medição e avaliação, com o propósito de fornecer um melhor entendimento dos processos para comparação de futuras atividades.

Para relatar a pesquisa, este artigo está estruturado da seguinte maneira: a Seção 2 apresenta o protocolo da RSL; a Seção 3 mostra os resultados dos trabalhos analisados, bem como uma visão geral dos artigos pesquisados; a Seção 4 descreve os resultados obtidos com a pesquisa e a Seção 5 exibe as conclusões acerca dos resultados.

\section{Protocolo da Revisão Sistemática}

Para elaborar a RSL, embasamos nossas ações nos métodos descritos por [Kitchenham \& Charters 2007], que abordam as etapas necessárias para a construção de uma boa Revisão Sistemática. Procuramos pesquisas mais atuais e relevantes realizadas sobre Pensamento Computacional, publicadas nos principais repositórios nacionais ligados à Sociedade Brasileira de Computação (SBC).

\subsection{Objetivo}

As metas desse trabalho foram definidas pelo protocolo GQM, que é um mecanismo para medição e avaliação, com o objetivo de fornecer um melhor entendimento dos processos para comparação de futuras atividades [Soligen \& Berghout 1999]. O Quadro 1 mostra os objetivos dessa RSL. 
VI Congresso Brasileiro de Informática na Educação (CBIE 2017)

Anais do XXVIII Simpósio Brasileiro de Informática na Educação (SBIE 2017)

Quadro 1. Objetivo conforme paradigma GQM.

\begin{tabular}{|l|l|}
\hline ANALISAR & Publicações científicas \\
\hline COM O PROPÓSITO DE & Caracterizar e analisar \\
\hline EM RELAÇÃO A & $\begin{array}{l}\text { Realizar um levantamento das pesquisas existentes nos sites } \\
\text { nacionais sobre o Pensamento Computacional. }\end{array}$ \\
\hline DO PONTO DE VISTA DOS & Pesquisadores \\
\hline NO CONTEXTO & Acadêmico \\
\hline
\end{tabular}

\subsection{Questão da Pesquisa}

Com base no objetivo supracitado, a principal questão de pesquisa apresentada é: Quais os principais Objetos de Aprendizagem são utilizados para realizar as atividades embasadas pelo Pensamento Computacional?

\subsection{Estratégias de Busca}

As buscas foram realizadas nos repositórios digitais relevantes na área de Informática na Educação no cenário brasileiro, tais como os relacionados ao Simpósio Brasileiro de Informática na Educação (SBIE), ao Workshop de Informática na Educação (WIE), ao Workshop de Educação em Informática (WEI), à Revista Brasileira de Informática na Educação (RBIE) e à Revista de Novas Tecnologias na Educação (RENOTE), desde o ano de 2012 até o ano atual, 2017. O idioma escolhido foi o português, por se tratar das pesquisas realizadas no Brasil.

\subsection{Expressão de Busca}

A string geral usada em todas os repositórios digitais foi ("pensamento computacional" OR “computational thinking") AND ("objetos de aprendizagem" OR "learning objects"), aplicados nos meta-dados (títulos, palavras-chave e resumo) dos artigos publicados.

\subsection{Critérios de Inclusão e Exclusão}

Com o intuito de responder à questão proposta na Subseção 2.2, foram inclusos e exclusos os artigos considerados relevantes e irrelevantes, respectivamente, à pesquisa, conforme os critérios descritos no Quadro 2.

\section{Quadro 2. Critérios de Inclusão e Exclusão}

\begin{tabular}{|l|l|}
\hline \multicolumn{1}{|c|}{ Critérios de Inclusão } & \multicolumn{1}{c|}{ Critérios de Exclusão } \\
\hline & CE1: Artigos de Revisão Sistemática de \\
CI1: Artigos de conferências ou revistas ligadas à & Literatura. \\
Sociedade Brasileira de Informática na Educação. & CE2: Pesquisas que não utilizam Objetos de \\
CI2: Publicados após o ano de 2012. & $\begin{array}{l}\text { Aprendizagem. } \\
\text { CE3: Trabalhos que não se aprofundam nos } \\
\text { resultados. }\end{array}$ \\
\hline
\end{tabular}

Após a aplicação da string de busca nos repositórios citados na Subseção 2.3, os resultados obtidos foram catalogados e encontram-se disponíveis no Quadro 3. Foram procurados artigos na Revista Brasileira de Informática na Educação, mas não foram encontrados trabalhos significativos para esta pesquisa. 
VI Congresso Brasileiro de Informática na Educação (CBIE 2017)

Anais do XXVIII Simpósio Brasileiro de Informática na Educação (SBIE 2017)

Quadro 3. Resultados Obtidos com a String de Busca.

\begin{tabular}{|l|c|c|c|c|}
\hline Repositório Digital & String de Busca & $\mathbf{1}^{\mathbf{0}}$ Filtro & $\mathbf{2}^{\mathbf{0}}$ Filtro & $\mathbf{3}^{\mathbf{o}}$ Filtro \\
\hline SBIE & 9 & 6 & 4 & 2 \\
\hline WIE & 26 & 23 & 15 & 8 \\
\hline WEI & 3 & 3 & 3 & 1 \\
\hline RENOTE & 1 & 1 & 1 & 1 \\
\hline TOTAL & 39 & 33 & 23 & 12 \\
\hline
\end{tabular}

Notamos pelo Quadro 3 que selecionamos inicialmente 39 artigos que continham a string de busca. Após uma leitura nos títulos e nos resumos, foram eliminados 6 estudos que não passaram no $1^{\mathrm{o}}$ filtro. Após a leitura da Introdução e da Metodologia, foi aplicado o $2^{\circ}$ filtro, visando retirar os trabalhos que não utilizavam Objetos de Aprendizagem em suas metodologias, sendo removidos 10 trabalhos.

As publicações com propostas empíricas ou com resultados pouco relevantes para utilização nessa pesquisa, foram retiradas. Para refinarmos a busca, foram lidos sequencialmente, os resumos, os resultados e as demais seções dos trabalhos. Em seguida, foi aplicado o $3^{\circ}$ filtro, onde conseguimos selecionar 12 estudos, que foram analisados e sintetizados para melhor visualizar as obras e assim responder à questão proposta nesta RSL.

\section{Trabalhos Analisados}

As informações foram extraídas para responder a questão de pesquisa desse trabalho. Isso permite classificar melhor os artigos e analisar sua importância para aplicação do PC no ambiente educacional. Embora não esteja listado entre os 12 artigos mais relevantes para esta obra, por se tratar de uma RSL, o trabalho publicado por [Zanetti et al. 2016] foi fundamental para nortear essa RSL, tendo em vista que mostrou e analisou as principais pesquisas de aplicação do PC no ensino de programação no cenário brasileiro.

[Andrade et al. 2013] propõem um conjunto de atividades lúdicas para alunos do ensino fundamental, onde abordam nove conceitos considerados essenciais para o desenvolvimento do PC nas escolas. As atividades foram os jogos Cara a Cara, Caça ao Tesouro e Organização de Festa. Depois de executados os trabalhos, foram discutidos junto aos alunos, os meios utilizados na realização dos jogos e foram analisadas as técnicas do PC para cumprir as tarefas de modo eficiente.

A apresentação de uma proposta metodológica para o ensino dos números binários para desenvolver o PC em crianças do $4^{\circ}$ ano foi abordada por [Campos et al. 2014]. As atividades foram fundamentadas na Computação Desplugada, onde com o uso de cartas, realizaram tarefas como conversão de números binários para decimal, de letras e palavras para números binários e vice-versa. Os resultados são expostos de forma quantitativa em vários critérios de avaliação adotados pelos pesquisadores, onde foram avaliados positivamente quanto ao incentivo do PC, mas que precisam de melhorias no método.

[Cavalcante et al. 2016] apresentam um estudo de caso qualitativo sobre as competências do PC exploradas em um curso de programação com a plataforma Code.Org, que é uma plataforma de ensino de programação online através de blocos. Foram verificadas várias atividades da plataforma, onde foram relacionadas as diversas competências do PC. Concluiu-se que os conceitos computacionais podem ser identificados durante a realização do curso, que podem ser aplicadas em atividades práticas com os alunos.

A avaliação de uma oficina de Scratch foi apresentada por [França \& Amaral 2013]. A pesquisa envolveu alunos de uma escola pública no Estado de Pernambuco, onde foram 
VI Congresso Brasileiro de Informática na Educação (CBIE 2017)

Anais do XXVIII Simpósio Brasileiro de Informática na Educação (SBIE 2017)

executadas atividades envolvendo os conceitos computacionais, como seleções e repetições, com o uso do Scratch. As avaliações mostraram que a ferramenta pode ser usada satisfatoriamente por estudantes da Educação Básica, de forma a estimular o pensamento computacional nos discentes.

A estratégia de ensino do PC incorporando técnicas Blended Learning foi explanada por [Gomes \& Melo 2013]. Essa técnica integra experiências de sala de aula com experiências online, combinando tecnologias e práticas que permeiam a aprendizagem significativa. Foi realizado um minicurso para estudantes do ensino médio para ensinar Lógica de Programação por meio do aplicativo Inventor for Android, que permite criar aplicativos para dispositivos móveis. A experiência em geral foi considerada boa por mais de $70 \%$ dos participantes, e $75 \%$ dos alunos responderam que tinham interesse em prosseguir os estudos na área de computação.

[Gomes et al. 2015] também explanaram sobre um jogo educativo, o The Foos, que foi avaliado por sob dois vieses: a avaliação formativa e a objetiva. O referido jogo foi criado para estimular o PC em crianças a partir de cinco anos de idade e consiste em guiar os personagens para realizar determinadas tarefas, usando blocos de instruções que representam ações. Os resultados mostraram que o software é de fácil uso, possui bons gráficos, é bem objetivo, motivador e possui um ambiente atrativo e divertido. Também mostraram que o jogo pode funcionar como um Objeto de Aprendizagem para estimular o Pensamento Computacional.

[Kologeski et al. 2016] descrevem um projeto interdisciplinar chamado Logicando, desenvolvido por estudantes e professores da Universidade Feevale, para desenvolver o raciocínio lógico nos alunos dos últimos anos do ensino fundamental das escolas locais. Foram realizadas oficinas que envolviam Lógica de Programação desde os fundamentos, até a o desenvolvimento de jogos, culminando com a realização de uma olimpíada de lógica. Durante a execução do projeto, foram usadas a plataforma Code.Org, o aplicativo MIT App Inventor, o Scratch e os jogos na rede social Teia: Navegática e Pantanal Escolar. Vale ressaltar que esses últimos recursos foram desenvolvidos pelos acadêmicos da Universidade Feevale. Os autores informam que o projeto Logicando ainda está em atividade e, que, apesar dos resultados começarem a aparecer agora, o retorno dos participantes tem sido bastante positivo.

Visando aumentar o interesse dos estudantes por algoritmos, [Pinho et al. 2016] descrevem a proposta e aplicação de uma atividade que introduz os conceitos elementares de algoritmos por meio de jogos de tabuleiro e de um jogo nomeado de Salve a Princesa, para alunos do quinto ano de escolas públicas. Foram aplicados conceitos de algoritmos para resolução de problemas, o que caracteriza habilidades específicas da Ciência da Computação. Os resultados foram mensurados por aplicação de testes antes e depois das atividades e foram considerados satisfatórios. As ações para desenvolver o PC foram alcançados, segundo a avaliação dos autores.

[Pinho et al. 2016] também mostram os resultados da atividade Caça ao Tesouro, onde aborda as premissas de busca de dados, utilizadas nos conceitos de Ciência da Computação. Para executar essa atividade, os autores usaram os conceitos da computação desplugada, que permitiu depois, desenvolver um jogo educativo digital. Foram avaliados os jogos nas formas clássica e digital, sendo comparados os resultados nas duas formas. Apesar da pouca vantagem apresentada pela plataforma digital, os autores não consideram sua superioridade.

[Ramos \& Teixeira 2015] analisam o resultado de um minicurso de Scratch voltado para o desenvolvimento de jogos para alunos do ensino médio. A experiência envolveu doze alunos em uma escola do Estado da Bahia e teve a duração de 16 horas. Durante o curso, 
VI Congresso Brasileiro de Informática na Educação (CBIE 2017)

Anais do XXVIII Simpósio Brasileiro de Informática na Educação (SBIE 2017)

houve produção de algoritmos e atividades práticas que envolveram a criação de jogos, sempre envolvendo os conceitos do PC. Os autores concluíram que foi possível produzir objetos digitais usando técnicas de programação, mesmo sem os conhecimentos prévios específicos em Ciências da Computação, e até mesmo em um espaço curto de tempo.

O uso da robótica para o ensino de lógica de programação e estimular o PC foi o tema de [Silva et al. 2016]. A metodologia apresentada pelos autores incluiu a utilização da tecnologia Lego Mindstorms e do Arduino como objetos de aprendizagem para auxiliar nas atividades. Primeiramente, foram apresentados os conceitos sobre Robótica e Programação, para em seguida, aplicarem atividades com o kit Lego. Para concluir o projeto, foi realizado um projeto com o Arduino. Observou-se que o uso da robótica educativa despertou o interesse científico nos alunos e os incentivou à resolução de problemas, que são essenciais para estimular o PC.

[Souza et al. 2016] abordam também a robótica como forma de desenvolver o PC nos alunos. Para isso, realizaram um curso de robótica com ênfase em Pensamento Computacional para o ensino médio nas Escolas SESI Paraíba, avaliando os efeitos do curso no desempenho de 729 estudantes do $1^{\circ}$ ano, nas disciplinas de robótica e base comum. Os discentes foram divididos em duas turmas: uma com uso do PC e outra, sem a aplicação dos conceitos do PC. A robótica foi aplicada com o uso do kit Lego. Os resultados estatísticos mostraram que $89 \%$ dos alunos que tiveram aplicação do PC obtiveram um desempenho melhor que os demais, demonstrando que o Pensamento Computacional aprimora o conhecimento dos estudantes.

\subsection{Visão Geral dos Artigos Analisados}

Apresentamos a seguir, uma visão geral dos artigos, com o objetivo de mostrar um resumo das atividades desenvolvidas e os objetos de aprendizagem utilizados nas pesquisas analisadas. Os dados estão expostos no Quadro 4.

Quadro 4. Lista de estudos analisados em ordem alfabética por autores

\begin{tabular}{|c|c|c|c|}
\hline Artigo & Autores & Atividade(s) & $\begin{array}{c}\text { Objeto(s) de } \\
\text { Aprendizagem(ns) }\end{array}$ \\
\hline A01 & [Andrade et al. 2013] & Atividades lúdicas & $\begin{array}{l}\text { Jogos Cara a Cara, Caça ao } \\
\text { Tesouro e Organização de Festa. }\end{array}$ \\
\hline A02 & [Campos et al. 2014] & $\begin{array}{l}\text { Computação Desplugada } \\
\text { para conversão de } \\
\text { números binários }\end{array}$ & Jogos de Cartas \\
\hline A03 & [Cavalcante et al. 2016] & $\begin{array}{l}\text { Ensino de Programação } \\
\text { através de blocos }\end{array}$ & $\begin{array}{l}\text { Plataforma de programação } \\
\text { Code.Org }\end{array}$ \\
\hline A04 & [França \& Amaral 2013] & $\begin{array}{l}\text { Conceitos de básicos e } \\
\text { avançados de Lógica de } \\
\text { Programação }\end{array}$ & Scratch \\
\hline A05 & [Gomes \& Melo 2013] & $\begin{array}{l}\text { Mincurso de Lógica de } \\
\text { Programação }\end{array}$ & Aplicativo Inventor for Android \\
\hline A06 & [Gomes et al. 2015] & Ensino de Lógica & Jogo The Foos \\
\hline A07 & [Kologeski et al. 2016] & $\begin{array}{l}\text { Projeto Logicando }- \\
\text { Ensino de Lógica de } \\
\text { Programação }\end{array}$ & $\begin{array}{l}\text { Plataforma Code.Org, aplicativo } \\
\text { MIT App Inventor, Scratch, } \\
\text { jogos na rede social Teia: } \\
\text { Navegática e Pantanal Escolar }\end{array}$ \\
\hline A08 & [Pinho et al. 2016] & $\begin{array}{l}\text { Conceitos elementares de } \\
\text { programação }\end{array}$ & $\begin{array}{l}\text { Jogos de Tabuleiros e o jogo } \\
\text { Salve a Princesa }\end{array}$ \\
\hline A09 & [Pinho et al. 2016] & Busca de dados & $\begin{array}{l}\text { Atividade Caça ao Tesouro, nas } \\
\text { formas clássica e digital }\end{array}$ \\
\hline
\end{tabular}


VI Congresso Brasileiro de Informática na Educação (CBIE 2017)

Anais do XXVIII Simpósio Brasileiro de Informática na Educação (SBIE 2017)

\begin{tabular}{|c|l|l|l|}
\hline A10 & {$[$ Ramos \& Teixeira 2015] } & $\begin{array}{l}\text { Produção de algoritmos e } \\
\text { criação de jogos }\end{array}$ & Scratch \\
\hline A11 & [Silva et al. 2016] & $\begin{array}{l}\text { Ensino de Lógica de } \\
\text { Programação }\end{array}$ & Lego Mindstorms e Arduino \\
\hline A12 & [Souza et al. 2016] & $\begin{array}{l}\text { Avaliar o efeito do PC nas } \\
\text { disciplinas de Robótica e } \\
\text { Base Comum. }\end{array}$ & Lego Mindstorms \\
\hline
\end{tabular}

\section{Resultados Obtidos}

Os artigos descritos foram selecionados a partir de processos de filtragens mencionados anteriormente na Seção 2. São resultados de trabalhos envolvendo aplicação do PC com a utilização de OAs no âmbito educacional brasileiro, em diversas situações e áreas do conhecimento. Apesar do crescimento de métodos e objetos para contribuir para a disseminação do PC, essas práticas ainda estão em ascensão no cenário educacional brasileiro. $\mathrm{O}$ número de material bibliográfico produzido tem crescido moderadamente nos últimos 5 anos, conforme podemos constatar na Figura 1.

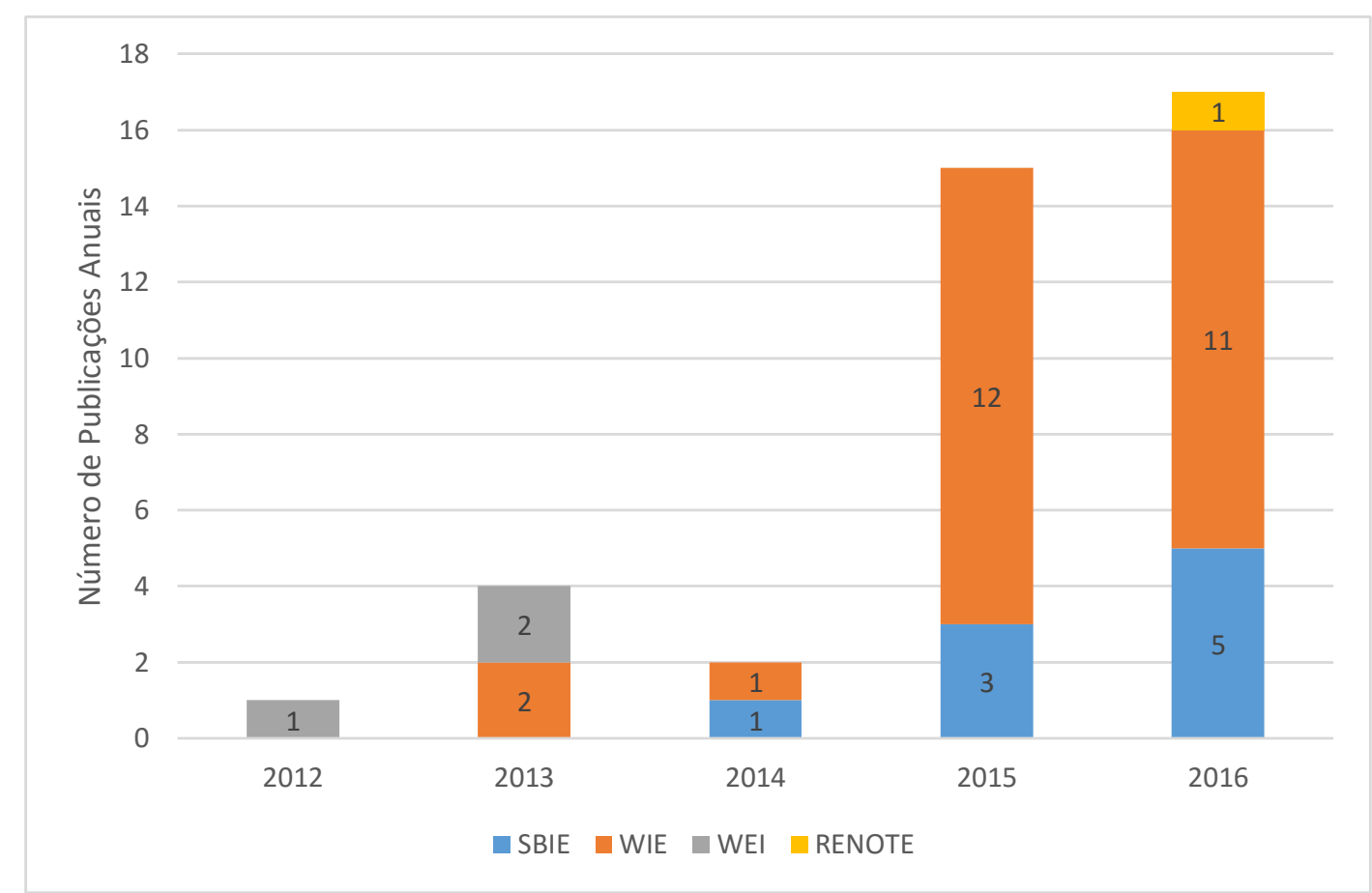

Figura 1. Número de Publicações sobre o Pensamento Computacional entre 2012 e 2016 nos principais repositórios digitais brasileiros sobre Informática na Educação.

Percebemos que os estudos visavam em sua maioria o desenvolvimento do raciocínio lógico, por meio das técnicas propostas por [Wing 2006], criadora do PC. Os alvos dos estudos nem sempre eram os estudantes de computação. Alguns projetos visavam introduzir discentes dos Ensinos Fundamental e Médio no contexto computacional. Na maioria dos trabalhos analisados, foram usadas pesquisas comparativas com a intenção de mensurar a influência do PC na aprendizagem de outras disciplinas, sempre chegando à conclusão que o $\mathrm{PC}$ pode realmente aprimorar o raciocínio lógico dos participantes. 
VI Congresso Brasileiro de Informática na Educação (CBIE 2017)

Anais do XXVIII Simpósio Brasileiro de Informática na Educação (SBIE 2017)

Os resultados obtidos mostram que as práticas mais usuais vão de ações mais concretas, como atividades lúdicas para estimular o raciocínio lógico, até atividades conceituais envolvendo programação. Os OAs utilizados nos artigos lidos compõem-se de jogos nas formas manuais e digitais, softwares educativos, kits de robótica e ambientes de programação. A Figura 2 detalha esses OAs e suas respectivas taxas de utilização nos artigos analisados nessa pesquisa.

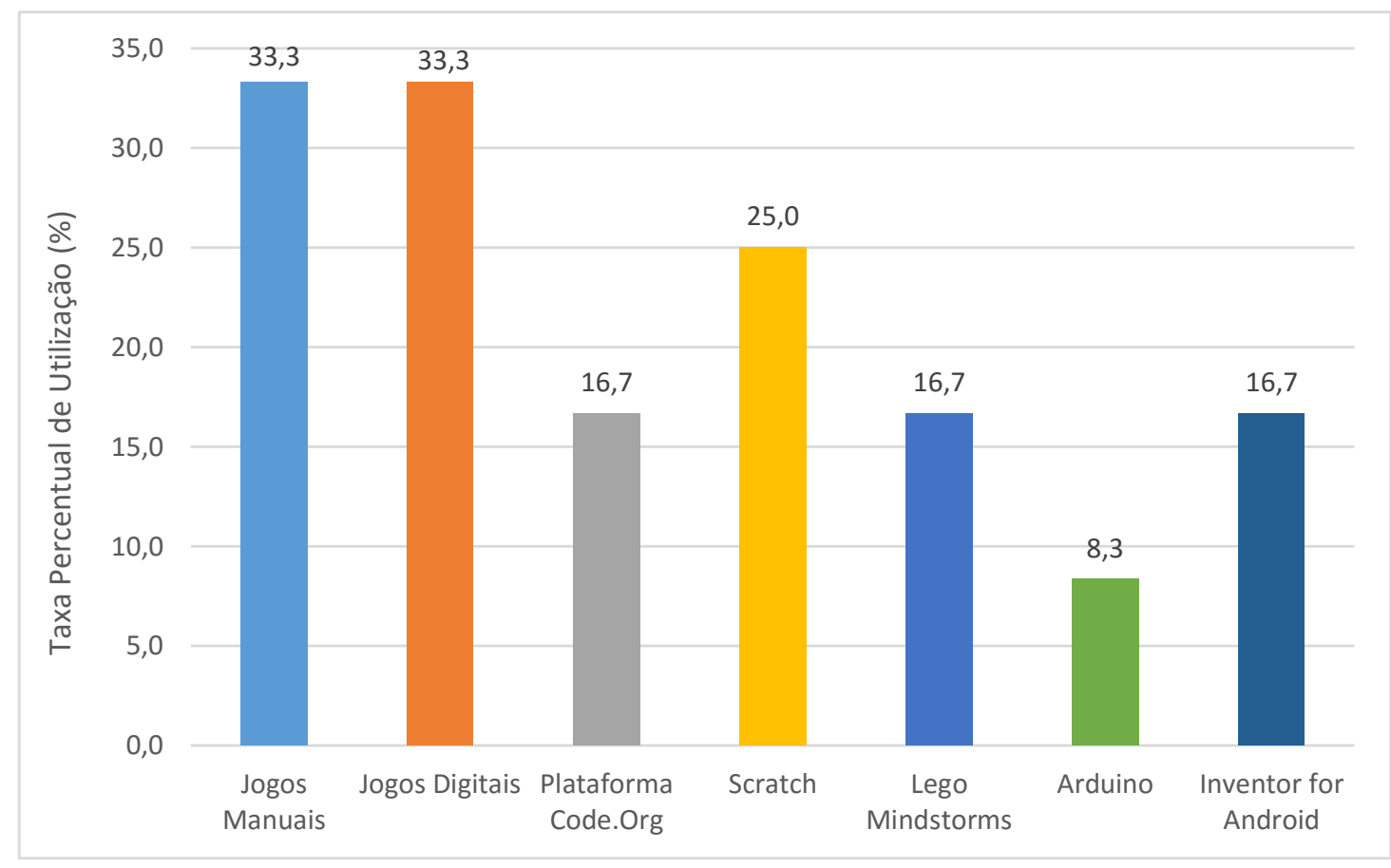

Figura 2. Objetos de Aprendizagem e respectivas Taxas Percentuais de Utilização nos trabalhos apresentados.

\section{Conclusões}

Esse estudo teve o objetivo de realizar um levantamento das pesquisas inerentes à aplicação do PC no meio educacional do Brasil e os OAs utilizados para esta finalidade. Foram pesquisados artigos publicados nos principais repositórios digitais relacionados à SBC, por meio da utilização da string de busca. Após a aplicação de filtros, foram selecionadas somente pesquisas que utilizavam PC com auxílio de OAs, a fim de analisar esses recursos, mesmo que não usassem explicitamente o termo Objeto de Aprendizagem e nem o definissem.

Empregar o PC realmente fez diferença no processo de ensino-aprendizagem, facilitando a absorção do conhecimento passado aos discentes. Essa pesquisa reforça ainda mais a importância da utilização dessas técnicas no meio educacional, mostrando que a computação pode ser uma grande ferramenta de apoio aos professores e alunos na busca pelo conhecimento.

Percebemos que as práticas analisadas incidiram sobre o aprendizado de Lógica. Contudo, a proposta do PC prevê a aplicação das técnicas nas mais diversas áreas do conhecimento acadêmico, tais como na Matemática, Biologia e demais ciências. Isso mostra o 
VI Congresso Brasileiro de Informática na Educação (CBIE 2017)

Anais do XXVIII Simpósio Brasileiro de Informática na Educação (SBIE 2017)

quanto ainda precisa ser feito nesse contexto no sistema educacional brasileiro, visando criar ou aprimorar metodologias de ensino embasadas por esta valiosa ferramenta.

Por meio desse trabalho, foi possível analisar também a importância que os OAs exercem no uso do PC. Além de contribuírem para absorção do conhecimento, estimularam os aprendizes a se interessarem mais pelo conteúdo apresentado pelos professores que usaram essas técnicas. Percebemos a variedade de ferramentas empregadas, seja de forma manual ou digital. Os OAs listados podem ser avaliados em estudos futuros para verificar suas eficácias em cenários diferentes dos que foram expostos, e assim, contribuir para disseminar o PC como ferramenta de apoio ao processo educacional.

\section{Referências}

Andrade, A.; Carvalho, T.;Silveira, J.; Cavalheiro, S.; Foss, L.; Marilza, Fleischmann, A.; Aguiar, M.; and Reiser, R. (2013). "Proposta de Atividades para o Desenvolvimento do Pensamento Computacional no Ensino Fundamental". In XIX Workshop de Informática na Escola. P.169-178.

Braga, Juliana e Menezes, Lilian (2014). "Introdução aos Objetos de Aprendizagem". In: BRAGA, Juliana Cristina (Org). Objetos de Aprendizagem - Volume 1: Introdução e Fundamentos. Santo André: Editora da UFABC.

Campos, G.; Cavalheiro, S.; Foss, L.; Pernas, A.; Piana, C.; Aguiar, M.; Bois, A.; and Reiser, R. (2014). "Organização de Informações via Pensamento Computacional: Relato de Atividade Aplicada ao Ensino Fundamental". In XX Workshop de Informática na Escola. P.390-399.

Cavalcante, A.; Costa, L.; and Araújo, A. 2016. "Um Estudo de Caso Sobre Competências do Pensamento Computacional Estimuladas na Programação em Blocos no Code.Org”. In V Congresso Brasileiro de Informática na Educação. P.1117-1126.

França, R. S. de; and Amaral, H. J. C. do. (2013). "Proposta Metodológica de Ensino e Avaliação para o Desenvolvimento do Pensamento Computacional com o Uso do Scratch". XIX Workshop de Informática na Escola. P.179-188.

Gomes, T. C. S.; and Melo, J. C. B. 2013. "O Pensamento Computacional no Ensino Médio: Uma Abordagem Blended Learning”. Workshop de Educação em Computação (WEI). Disponível em < http://www.lbd.dcc.ufmg.br/colecoes/wei/2013/0033.pdf> Acesso em 23/04/2017 às $00: 35 h$.

; Barreto, P. P.; Lima, I. R. A.; and Falcão, T. P. 2015. “Avaliação de um Jogo Educativo para o Desenvolvimento do Pensamento Computacional na Educação Infantil". In Anais do Congresso Brasileiro de Informática na Educação. P.1349-1358.

Kitchenham, Barbara e Charters, Stuart. (2007). "Guidelines for performing Systematic Literature Reviews in Software Engeneering". Keele University and Duham University.

Kologeski, A. L.; Silva, C. G.; Barbosa, D. N. F.; Mattos, R. R.; and Miorelli, S. T. "Desenvolvendo o Raciocínio Lógico e Pensamento Computacional: Experiências no Contexto do Projeto Logicando". 2016. Revista Novas Tecnologias da Educação. V.14, N.2, dez.

Pinho, G.; Weisshahn, Y.; Cavalheiro, S.; Reiser, R.; Piana, C.; Foss, L.; Aguiar, M.; and Bois, A. D. 2016. "Pensamento Computacional no Ensino Fundamental: Relato de Atividade de Introdução a Algoritmos". In XXII Workshop de Informática na Escola. P.261-270. 
; Weisshahn, Y.; Cavalheiro, S.; Cavalheiro, G.; and Piana, C. F. de B. 2016. "Proposta de Jogo Digital para Dispositivos Móveis: Desenvolvendo Habilidades do Pensamento Computacional”. In XXVII Simpósio Brasileiro de Informática na Educação. P.100-109.

Ramos, F. O. and Teixeira, L. da S. 2015. "Significação da Aprendizagem Através do Pensamento Computacional no Ensino Médio: Uma Experiência com Scratch”. In XXI Workshop de Informática na Escola. P.217-226.

Silva D. P. da; Sidnei, S. S.; Jesus, A. M. de; and Silva, C. E. P. 2016. "Aplicação de Robótica na Educação de Forma Gradual para o Estímulo do Pensamento Computacional". In V Congresso Brasileiro de Informática na Educação. P.1188-1197.

Soligen, R. e Berghout, E., (1999). "The goal/question/metric method - A pratical guide for quality improvement of software development".

Souza, I. M. L.; Rodrigues, R. S.; and Andrade, W. L. 2016. "Explorando Robótica com Pensamento Computacional no Ensino Médio: Um Estudo sobre seus Efeitos na Educação”. In XXVII Simpósio Brasileiro de Informática na Educação. P.490-499.

Wing, Jeannette (2006). "Computational Thinking”. Communications of the ACM. V.49, n.3, p.33-35, mar.

Zanetti, H. A. P.; Borges, M. A. F.; Ricarte, I. L. M. 2016. "Pensamento Computacional no Ensino de Programação: Uma Revisão Sistemática da Literatura Brasileira". In XXVII Simpósio Brasileiro de Informática na Educação. P.21-30. 